\title{
The frequency of cytogenetic abnormalities in idiopathic oligospermia and azoospermia infertile men in Chaharmahal and Bakhtiari province: a cross-sectional study
}

\author{
Hamideh Jafari-Ghahfarrokhi ${ }^{(\mathbb{D}}$, Delnya Gholami ${ }^{1}$, Mohammad Rajaie Esfahani ${ }^{2}$, Hossein Teimori ${ }^{*{ }^{*}}$ \\ ${ }^{1}$ Cellular and Molecular Research Center, Basic Health Sciences Institute, Shahrekord University of Medical Sciences, Shahrekord, \\ Iran. \\ ${ }^{2}$ Department of Urology, Shahrekord University of Medical Sciences, Shahrekord, Iran.
}

*Corresponding Author: Dr. Hossein Teimori, Associate Professor, Head of Medical Genetics, Shahrekord University of Medical Sciences, Tel: +98-38-33346691 Email: hteimori@skums.ac.ir

\begin{abstract}
Background and aims: Infertility is one of the main health issues in families worldwide. In addition, there is a complex correlation between genetics and infertility and chromosomal abnormalities are found in $8 \%$ of infertile males. The aim of this study was to determine the frequency of cytogenetic abnormalities among idiopathic oligospermia and azoospermia infertile men who were treated in Chaharmahal and Bakhtiari province.

Methods: In this cross-sectional study, the records of a total of 100 participants were evaluated retrospectively. The patients who were under careful physical and para-clinical (i.e., hormonal, ultrasound, and spermiogeram) examinations were enrolled in the study. Chromosomal analysis was carried out on the cultures of peripheral blood lymphocytes by Giemsa (G) banding. Eventually, 10 well-spread metaphases were analyzed by G-banding.

Result: The chromosome abnormality frequency, the numerical type, and the structural type were $13 \%, 3 \%$, and $10 \%$, respectively. Among patients with azoospermia, two cases had Klinefelter syndrome with karyotype.

Conclusion: This study demonstrated that structural abnormalities are more prevalent than numerical abnormalities in infertile men who were treated in Chaharmahal and Bakhtiari province. This indicates the importance of cytogenetic examination and the relevance of its achievements to the patient's management in infertility clinics. Therefore, the cytogenetic test is proposed for infertile men, in particular in those who endure azoospermia.

Keywords: Chromosomal abnormality, Male infertility, Oligospermia, Azoospermia
\end{abstract}

Received: 15 May 2019, Accepted: 13 July 2019, ePublished: 28 February 2020

\section{Introduction}

Infertility is considered as one of the main health issues in families worldwide, which is the inability to conceive after one year of unprotected intercourse (1). Overall, male infertility is attributed to several factors. Genetic factors involved in male fertility include chromosome structural problems and genetic syndromes that lead to genetic disorders in infertile men. Around 30\%-40\% of men referring to infertility centers have a genetic abnormality $(2,3)$. In addition, $5 \%-10 \%$ of infertile men suffer from oligospermia and 15\%-20\% of those with azoospermia have a genetic abnormality. Chromosome abnormalities are much more common in men with azoospermia and oligospermia compared to the general population. It was reported that abnormalities in sex chromosomes were higher in azoospermia group than in the oligospermia group (4). An inverse correlation was demonstrated between chromosome abnormalities and the amount of sperm in the semen. However, no data are available regarding the exact mechanism through which chromosome abnormalities lead to infertility. It has been stipulated that dispersed chromatin interactions alongside meiosis reduce sperm production (5). Further, the type of chromosome abnormalities depends on suffering from oligospermia or azoospermia (5). In the general population, aneuploidy (10.8\%) represents the most frequent cause of male infertility and Klinefelter syndrome is the most prevalent abnormality. The incidence of this syndrome is around 5\% in infertile men with severe oligospermia and $10 \%$ in those with azoospermia $(3,5)$.

(C) 2020 The Author(s); Published by Shahrekord University of Medical Sciences. This is an open-access article distributed under the terms of the Creative Commons Attribution License (http://creativecommons.org/licenses/by/4.0), which permits unrestricted use, distribution, and reproduction in any medium, provided the original work is properly cited. 
The Y chromosome microdeletions are the second genetic cause of male infertility after Klinefelter syndrome (6). The frequency of $\mathrm{Y}$ chromosome microdeletions was $6.17 \%$ in Chaharmahal and Bakhtiari Province (7). Generally, men with karyotype 47, XYY are fertile although this karyotype is much frequently observed in infertile men. The sexual reverse syndrome is another genetic cause of male infertility in men with azoospermia indicating the karyotype 46,XX. The frequency of chromosome polymorphisms in infertile men is $7.9 \%$ (8) and Y chromosome polymorphisms have been found in people with azoospermia and oligospermia. Furthermore, the variants on the $\mathrm{Y}$ chromosome are developed due to the variants in the close region of the long arm that includes the heterochromatin region. The percentage of these variables is $3.4 \%$ and $27.3 \%$ for yqh+ and yqh-, respectively. Moreover, the long Y chromosome is associated with a high risk of miscarriage (9). Long heterochromatin $9 \mathrm{qh}+$ is also a well-known polymorphism with an incidence rate of $6 \%-8 \%(9,10)$. Considering the significance of detecting the type of cytogenetic problem (oligospermia and azoospermia) and the high frequency of cytogenetic disorders among infertile men, the primary aim of this study was to investigate the causes of azoospermia and oligospermia cytogenetically using the karyotype technique in infertile men. Then, it was attempted to evaluate the frequency of Y chromosome polymorphisms in infertile men who were treated in Chaharmahal and Bakhtiari province.

\section{Materials and Methods}

In this cross-sectional study, the blood samples of 100 infertile men with oligospermia and azoospermia were taken after they referred to the health care centers of Hajar hospital and diagnosed by a physician according to the test result. Sixty patients were assigned to azoospermia group and the remaining cases were included in the oligospermia group.

Couples' failure to conceive after at least 1 year having sex without using contraceptive methods was considered the criterion for infertility. The patients who were under careful physical and para-clinical (i.e., hormonal, ultrasound, and spermiogeram) examinations were enrolled in the study. The patients with non-genetic causes of infertility, including those acquiring mumps in childhood, as well as patients with varicocele (including those who had undergone surgery or had not yet been treated) were excluded from the study. Then, they were grouped according to the sperm count. Oligospermia refers to a condition where the concentration of the sperm (sperm count) in the semen is low and less than 20 million per milliliter of semen and azoospermia refers to a considerable lack of sperm in the fluid semen (11). The peripheral blood was taken from each patient and introduced into a $5 \mathrm{~mL}$ tube containing heparin sodium. Next, blood cell culture and cell harvest were conducted in the first and second phases of the study, respectively, followed by isolating blood lymphocytes from the remaining cell materials. In the third phase, a slide was taken from the sediments and several slides were prepared for each patient. Then, the slides were incubated for four weeks. In the fourth phase ( $G$ banding), the samples were stained with Giemsa and the slides were mounted as well. In the fifth phase, the slides were examined and then chromosome analysis was conducted on the culture of peripheral blood lymphocytes. Additionally, chromosome abnormalities were recorded based on the International System for Human Cytogenomic Nomenclature guideline (2013). Finally, 10 metaphases were analyzed by cytovision software and 20 metaphases were counted as well.

\section{Data Analysis}

The percentage of patients with chromosome abnormalities in azoospermia and oligospermia groups was taken in the present study.

\section{Results}

In this study, 100 patients were evaluated, including those suffering from azoospermia $(\mathrm{n}=60)$ and oligospermia $(n=40)$. The mean age of the patients was $32.7 \pm 0.79$ (within the range of 21-47 years). In addition, the prevalence of chromosome abnormalities was $13 \%$ encompassing $3 \%$ and $10 \%$ for numerical and structural abnormalities, respectively (Table 1). Among patients with azoospermia, two cases had Klinefelter syndrome with karyotype 47,XXY (Figure 1), one had sexual inversion with karyotype 46,XX, one had karyotype 47,XYY (Figure 2), four patients had increased long arm of yqh+ chromosome, and one had increased $\mathrm{q}$ arm with karyotype ins1(q11.2)(qh+). Among patients with oligospermia, three cases had an increased long arm of chromosome 13 with karyotype 46,XY,13ph+ and one had karyotype 46,XY,14ph+.

\section{Discussion}

The prevalence of chromosome abnormalities among men with azoospermia and oligospermia was reported as $14.2 \%$ and $6.5 \%$ within the ranges of $10 \%-15 \%$ and $5 \%-7 \%$, respectively. In the current study, chromosome

Table 1. Chromosome Abnormalities Observed Among the Studied People

\begin{tabular}{lccc}
\hline Karyotype & $\begin{array}{c}\text { Azoospermia } \\
\text { No. }(\%)\end{array}$ & $\begin{array}{c}\text { Oligospermia } \\
\text { No. }(\%)\end{array}$ & $\begin{array}{c}\text { Total } \\
\text { No. }(\%)\end{array}$ \\
\hline $47, \mathrm{XXY}$ & $2(0.03)$ & & $2(0.02)$ \\
$46, \mathrm{XX}$ & $1(0.01)$ & & $1(0.01)$ \\
$47, \mathrm{XYY}$ & $1(0.01)$ & & $1(0.01)$ \\
Yqh+ & $4(0.06)$ & & $4(0.04)$ \\
$\operatorname{Ins1}(\mathrm{q} 11.2)(\mathrm{qh}+)$ & $1(0.01)$ & $3(0.07)$ & $1(0.01)$ \\
$46, \mathrm{XY}, 13 \mathrm{ph}+$ & & $1(0.02)$ & $1(0.03)$ \\
$46, \mathrm{XY}, 14 \mathrm{ph}+$ & & & \\
\hline
\end{tabular}




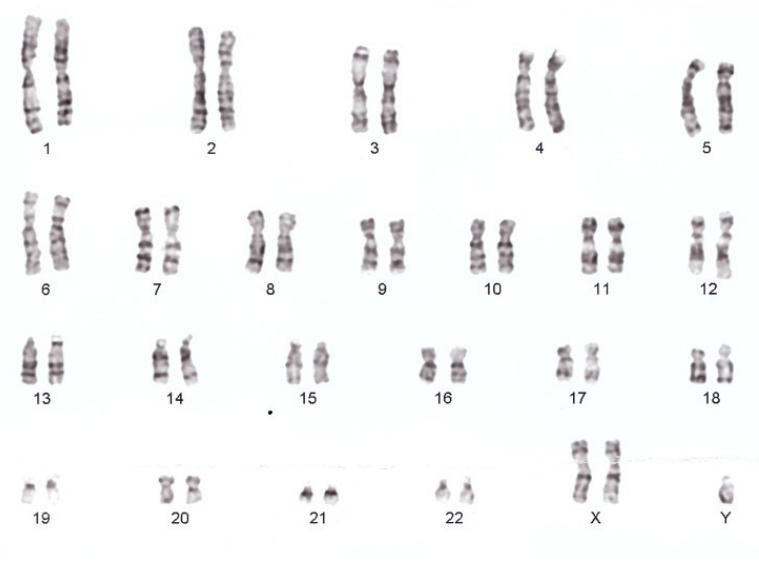

Figure 1. Klinefelter Karyotype A Man With Karyotype 47,XXY.

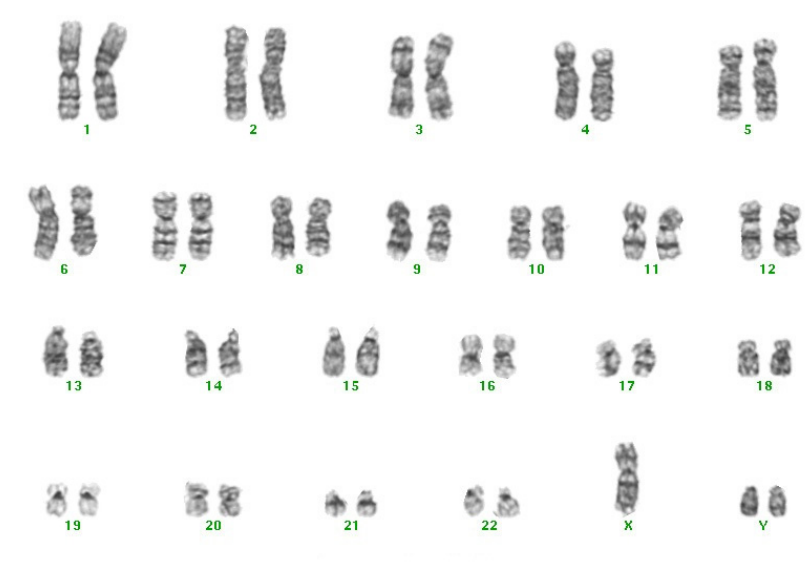

Figure 2. Karyotype XYY A Man With Karyotype 47, XYY.

abnormalities in the azoospermia and oligospermia groups of investigated cases were $15 \%$ and $10 \%$, respectively.

In the general population, aneuploidy $(10.8 \%)$ represents the most frequent cause of male infertility and Klinefelter syndrome is the most frequent abnormality (5). In the present study, the total prevalence of Klinefelter syndrome was $2 \%$ and its prevalence was $3 \%$ in the azoospermia group. Ferlin et al reported that the incidence of Klinefelter syndrome in infertile men was very high and it was about $5 \%$ and $10 \%$ in infertile men with severe oligospermia and azoospermia, respectively (12). This disease occurs in one per 1000 live births in the general population of men (13).

$\mathrm{Y}$ chromosome polymorphisms were observed in people with azoospermia and severe oligospermia as well. In the present study, $6 \%$ of men with azoospermia and $4 \%$ of all men had yqh+ chromosome. Further, long and short $Y$ chromosomes are found in infertile people. Y chromosome variants are frequently observed as yqh+ and yqh- with $3.4 \%$ and $27.3 \%$ frequency, respectively. Genest and
Genest reported that the short $\mathrm{Y}$ chromosome was not associated with the high risk of miscarriage. The presence of $Y$ chromosome variants in infertile men is a controversial issue that needs further investigation (14).

Generally, men with karyotype 47, XYY are fertile, but this karyotype is frequently found in infertile men. ElDahtory et al studied four men with azoospermia who referred to the genetic clinic. The chromosome analysis of peripheral blood lymphocytes demonstrated karyotype $47, \mathrm{XYY}$ and the presence of an additional Y chromosome was confirmed by the fluorescence in situ hybridization technique (15). In the present study, $1.6 \%$ of men with azoospermia and $1 \%$ of all men had karyotype $47, \mathrm{XYY}$. The sexual reverse is another genetic cause of infertility in men with azoospermia that occurs in 1.6 of these cases.

Abusheikha et al reported a 28-year-old man with normal phenotype with karyotype 46,XX. In addition, the PCR technique confirmed the presence of the sex-determining region $\mathrm{Y}$ (SRY) gene on one of the $\mathrm{X}$ chromosomes. This case was the sixth reported one to date. In the present study, $1.6 \%$ of men with azoospermia and $1 \%$ of all men had karyotype 47,XX. Men with the karyotype 46,XX are included in men with azoospermia. The most frequent cause of this abnormality occurs due to crossing over between Xp and Up along father meiosis (10). Therefore, the SRY gene localizes on the X chromosome and is present in the karyotype (SRY+XX males), but such patients have azoospermia.

Balanced autosomal translocations and male infertility were found to be associated in men with severe oligospermia and azoospermia. The mechanism through which such chromosome abnormalities cause infertility has not yet been exactly known. Moreover, the karyotype studies of the spermatozoa of 37 heterozygous double translocations demonstrated that $19 \%-77 \%$ of spermatozoa were unbalanced. When seeking to determine the genetic basis of male infertility, it should be noted that about 59\% of the observed translocations in infertile men involve acrocentric chromosomes, which highlights the role of these chromosomes in the reduction of male fertility (5). The prevalence of Robertsonian translocation in infertile men is only $0.8 \%$, which is nine times higher than its prevalence in the general population (8). In the current study, $10 \%$ of men with oligospermia and $4 \%$ of all men had acrocentric chromosome heteromorphism. Guichaoua et al emphasized the association between the involvement of acrocentric chromosomes in translocation carriers among infertile men and the severity of the spermatogenic defect (16). It was suggested that balanced translocations interfere with the pairing of normal chromosomes and their separation during meiosis 1 and provide conditions to create unbalanced gametes, thus giving birth to unbalanced and abnormal neonates. Another hypothesis is that the expression regulations of potential autosomal genes 
involved in gametogenesis are likely to be eliminated by chromosome breakpoints. The evaluation of the association between chromosome breaks and male infertility revealed the relationship between non-accidental chromosome breaks and infertility.

Additionally, XX male syndrome is one of the causes of male infertility. Men with karyotype 46,XX are infertile cases with normal female chromosomes (17). This occurs in one per 9000-20000 cases. Similarly, the majority of such patients acquired this disease as sporadic and had no family history of this disease. This disease is due to the exchange of a piece of $Y$ chromosome short arm that codes the testis-determining factor gene on the $\mathrm{X}$ chromosome. So far, two types of this syndrome have been identified, including positive and negative Y DNA. Positive Y DNA occurs due to the translocation of $\mathrm{XY}$ with a low risk of repeated incidence. Men with negative Y DNA develop this syndrome due to a mutation with a high risk of incidence. In addition, all men with such chromosome patterns have azoospermia, which is due to the lack of the long arm of the $\mathrm{Y}$ chromosome that includes the azoospermia factor gene $(10,18,19)$.

Common cytogenetic polymorphisms identified by G-banding, considered to be heteromorphism, represent another cause of male infertility and include heterochromatin regions of $16,9,1$, and $\mathrm{Y}$ chromosomes $(10,13)$. However, this diagnosis for such patients is highly improbable due to the presence of the normal male outer reproductive tract.

\section{Conclusion}

In the current study, the chromosome abnormality frequency, the numerical type, and the structural type were $13 \%, 3 \%$, and $10 \%$, respectively. These findings can be attributed to male infertility, but not all cases are necessarily the main cause of infertility. More clearly, each of these symptoms may be observed in normal people. An important implication of this study is that cytogenetic analysis should be conducted on these patients before studying $\mathrm{Y}$ chromosome partial deletions because most infertile people who have no $\mathrm{Y}$ chromosome partial deletions are likely to have a cytogenetic problem.

Conflict of Interests

The authors declare that there is no conflict of interests.

Acknowledgments

This study was supported by the Research and Technology Deputy of Shahrekord University of Medical Sciences (Code number: 1733).

\section{Ethical Approval}

The study protocol was approved by the Ethics Committee of Shahrekord University of Medical Sciences (the ethics code of IR.SKUMS.REC.15-7-92).
Authors Contribution

H J-G : designed the study, carried out data collection.

D G: participation in analysis and carried out data collection.

M R-E: contributed to collected all of samples.

H T:contributed to study design and manuscript drafting.

all authors approved the final version manuscript.

\section{Funding/Support}

This study was supported by the Research and Technology Deputy of Shahrekord University of Medical Sciences.

\section{Informed Consent}

The patients filled out a questionnaire of medical information and provided informed consent for participating in the study in Shahrekord Medical University.

\section{References}

1. Zaimy MA, Kalantar SM, Sheikhha MH, Jahaninejad T, Pashaiefar $\mathrm{H}$, Ghasemzadeh J, et al. The frequency of $\mathrm{Yq}$ microdeletion in azoospermic and oligospermic Iranian infertile men. Iran J Reprod Med. 2013;11(6):453-8.

2. Akgul M, Ozkinay F, Ercal D, Cogulu O, Dogan O, Altay B, et al. Cytogenetic abnormalities in 179 cases with male infertility in Western Region of Turkey: report and review. J Assist Reprod Genet. 2009;26(2-3):119-22. doi: 10.1007/s10815-009-92968 .

3. Azimi C, Khaleghian M, Farzanfar F. Cytogenetic studies among Iranian infertile men: the first 20-year long-term report. Afr J Biotechnol. 2012;11(37):8973-8.

4. Lissitsina J, Mikelsaar R, Punab M. Cytogenetic analyses in infertile men. Arch Androl. 2006;52(2):91-5. doi: 10.1080/01485010500316030.

5. Nie H, Lu G. Long Y chromosome is not a fetal loss risk. J Assist Reprod Genet. 2011;28(2):151-6. doi: 10.1007/s10815-0109497-1.

6. Krausz C, Hoefsloot L, Simoni M, Tüttelmann F. EAA/EMQN best practice guidelines for molecular diagnosis of Y-chromosomal microdeletions: state-of-the-art 2013. Andrology. 2014;2(1):519. doi: 10.1111/j.2047-2927.2013.00173.x.

7. Gholami D, Jafari-Ghahfarokhi H, Nemati-Dehkordi M, Teimori $\mathrm{H}$. Y chromosome microdeletions frequency in idiopathic azoospermia, oligoasthenozoospermia, and oligospermia. Int J Reprod Biomed (Yazd). 2017;15(11):703-12.

8. O'Flynn O'Brien KL, Varghese AC, Agarwal A. The genetic causes of male factor infertility: a review. Fertil Steril. 2010;93(1):1-12. doi: 10.1016/j.fertnstert.2009.10.045.

9. Belangero SI, Christofolini DM, Bianco B, Gava MM, Wroclawski ER, Barbosa CP. Male infertility related to an aberrant karyotype, 46,XY,9ph,9qh+. Fertil Steril. 2009;91(6):2732.e1-3. doi: 10.1016/j.fertnstert.2008.10.057.

10. Abusheikha N, Lass A, Brinsden P. XX males without SRY gene and with infertility. Hum Reprod. 2001;16(4):717-8. doi: 10.1093/humrep/16.4.717.

11. Vasan SS. Semen analysis and sperm function tests: how much to test? Indian J Urol. 2011;27(1):41-8. doi: 10.4103/09701591.78424.

12. Ferlin A, Arredi B, Foresta C. Genetic causes of male infertility. Reprod Toxicol. 2006;22(2):133-41. doi: 10.1016/j. reprotox.2006.04.016.

13. Dong Y, Jiang YT, Du RC, Zhang HG, Li LL, Liu RZ. Impact of chromosomal heteromorphisms on reproductive failure and analysis of 38 heteromorphic pedigrees in Northeast China. J Assist Reprod Genet. 2013;30(2):275-81. doi: 10.1007/ s10815-012-9910-z.

14. Genest $\mathrm{P}$, Genest $\mathrm{FB}$. The influence of the length of the human $\mathrm{Y}$ 
chromosome on spontaneous abortions. A prospective study in family lines with inherited polymorphic Y chromosomes. Ann Genet. 1985;28(3):143-8.

15. El-Dahtory F, Elsheikha HM. Male infertility related to an aberrant karyotype, 47,XYY: four case reports. Cases J. 2009;2(1):28. doi: 10.1186/1757-1626-2-28.

16. Guichaoua MR, Quack B, Speed RM, Noel B, Chandley AC, Luciani JM. Infertility in human males with autosomal translocations: meiotic study of a 14;22 Robertsonian translocation. Hum Genet. 1990;86(2):162-6. doi: 10.1007/ bf00197698.

17. Coşar R, Demirci H, Sayın Kocakap DB, Çiftçi Ö, Eren Kavak
EE. 46, XX Sry(+) male sexual differentiation disorder with metabolic syndrome: a case report. Turk J Endocrinol Metab. 2014;1:28-30. doi: 10.4274/tjem.2340.

18. Thangaraj K, Gupta NJ, Pavani K, Reddy AG, Subramainan S, Rani DS, et al. Y chromosome deletions in azoospermic men in India. J Androl. 2003;24(4):588-97. doi: 10.1002/j.19394640.2003.tb02710.x.

19. Yu XW, Wei ZT, Jiang YT, Zhang SL. Y chromosome azoospermia factor region microdeletions and transmission characteristics in azoospermic and severe oligozoospermic patients. Int J Clin Exp Med. 2015;8(9):14634-46. 